BULLETIN (New Series) OF THE AMERICAN MATHEMATICAL SOCIETY

Volume 36, Number 1, January 1999, Pages 75-93

S 0273-0979(99)00771-5

\title{
FINITE SIMPLE GROUPS WHICH PROJECTIVELY EMBED IN AN EXCEPTIONAL LIE GROUP ARE CLASSIFIED!
}

\author{
ROBERT L. GRIESS JR. AND A. J. E. RYBA
}

\begin{abstract}
Since finite simple groups are the building blocks of finite groups, it is natural to ask about their occurrence "in nature". In this article, we consider their occurrence in algebraic groups and moreover discuss the general theory of finite subgroups of algebraic groups.
\end{abstract}

\section{INTRODUCTION}

Group character theory classifies embeddings of finite groups into classical groups. No general theory classifies embeddings into the exceptional complex algebraic group, i.e., one of $G_{2}(\mathbb{C}), F_{4}(\mathbb{C}), E_{6}(\mathbb{C}), E_{7}(\mathbb{C}), E_{8}(\mathbb{C})$. For exceptional groups, special methods seem necessary. Since the early $80 \mathrm{~s}$, there have been efforts to determine which central extensions of finite simple groups embed in an exceptional group. For short, we call this work a study of projective embeddings of finite simple groups into exceptional groups. Table PE on page 84 contains a summary.

The classification program for finite subgroups of complex algebraic groups involves both existence of embeddings and their classification up to conjugacy. We have just classified embeddings of $S z(8)$ into $E_{8}(\mathbb{C})$ (there are three, up to $E_{8}(\mathbb{C})$ conjugacy), thus settling the existence question for projective embeddings of finite simple groups into exceptional algebraic groups. The conjugacy part of the program is only partially resolved.

The finite subgroups of the smallest simple algebraic group $P S L(2, \mathbb{C}$ ) (up to conjugacy) constitute the famous list: cyclic, dihedral, Alt $4, S_{4} m_{4}, A t_{5}$. This list has been associated to geometry, number theory, and Lie theory in several ways. McKay's correspondence between these groups and the Cartan matrices of types $A, D$ and $E$ and his related tensor product observations $[\mathrm{McK}]$ are provocative. For the exceptional algebraic groups, theories of Kostant, Springer and Serre have called attention to particular finite simple subgroups. A good list of finite subgroups should help us understand the exceptional groups better.

\section{TABLE OF CONTENTS}

0 . Introduction

1. General theory of homomorphisms of finite groups to algebraic groups.

2. Classification of finite quasisimple subgroups.

Received by the editors April 13, 1998, and in revised form May 19, 1998, and October 16, 1998.

1991 Mathematics Subject Classification. Primary 17Bxx, 20Bxx, 20Cxx, 20Dxx, 20Exx, 22Exx. 
3. Table of projective embeddings of finite simple groups into exceptional algebraic groups.

4. Sketch of the computer work on hard embeddings.

5. History of classifications of finite subgroups of exceptional groups.

6. Related classifications.

7. References.

\section{ACKNOWLEDGEMENTS}

The first author thanks the NSF for its support under grant number DMS9623038.

The second author thanks the NSA for its support under grant number MDA90497-1-0043 and thanks the Department of Mathematics at the University of Michigan for computer access.

\section{LisT OF NOTATIONS}

$Z(G)$

$C(H)=C_{G}(H)$

$N(H)=N_{G}(H)$

$x^{y}=y^{-1} x y$

$[x, y]=x^{-1} y^{-1} x y$

$G^{\prime}$

$H \circ K$

$H . K$

$H: K$

$H \cdot K$

$m$

$m^{n}$

Sym $_{n}$

$\mathbb{F}_{p}$

$\overline{\mathbb{F}}_{p}$

$G L, S L$

$P G L, P S L$

$A G L$

$O, S O$

Spin, HSpin

$S z\left(2^{2 k+1}\right)$

$W(\Phi)$

$G^{0}$

$\operatorname{Emb}(F, G), \operatorname{Proj} \operatorname{Emb}(F, G)$ the center of the group $G$

the centralizer of the subset $H$ of $G$

the normalizer of the subset $H$ of $G$

the conjugate of $x$ by $y$

the commutator of group elements $x$ and $y$

the commutator subgroup of the group $G$

a central product of groups $H$ and $K$

an extension of the group $K$ by $H$, i.e., a group

$G$ with normal subgroup $N \cong H$ such that

$G / N \cong K$

a split extension of shape H.K (example: $31: 30$

denotes a group with normal cyclic subgroup

of order 31 and cyclic quotient of order 30 )

a nonsplit extension of shape $H . K$ (example:

the group $S L(2,5)$ of order 120 is of shape $2 \cdot A l t_{5}$, the double cover of the simple group of order 60 )

the integer $m$ stands for a cyclic group of order $m$ a finite group which is the direct product of $n$ copies of a cyclic group of order $m$ (example: $2^{2}$ is the direct product of two groups of order 2)

the symmetric group on $n$ objects

the field of prime order $p$

the algebraic closure of the field of prime order $p$

the general, special linear group

the projective general, special linear group

the affine general linear group

the orthogonal, special orthogonal group

the spin, half spin groups

the Suzuki simple group defined over the field of $2^{2 k+1}$ elements, $k \geq 1$

the Weyl group of a root system of type $\Phi$

the connected component of the identity in the algebraic group $G$

the set of embeddings, projective embeddings, resp., of the finite group $F$ in the group $G$ 


\section{General theory of homomorphisms of finite groups to algebraic GROUPS}

The problem of classifying finite dimensional complex representations of a finite group is an old one. Given a finite group $F$, an $n$-dimensional representation is a homomorphism from $F$ to a general linear group $G L(n, \mathbb{C})$. Two $n$-dimensional representations are equivalent if they are conjugate under $G L(n, \mathbb{C})$. Classical character theory is considered a good solution to the problem of classifying representations. (Definition: the character of a representation $F \rightarrow G L(n, \mathbb{C})$ is the function which assigns the trace of the representing matrix to a group element. A character is a class function since conjugate matrices have the same trace. Two representations are equivalent iff they have the same character.)

Consider the symmetric group of degree 4 which has character table

\begin{tabular}{rrrrr}
$1^{4}$ & $1^{2} 2^{1}$ & $2^{2}$ & $1^{1} 3^{1}$ & $4^{1}$ \\
\hline 1 & 1 & 1 & 1 & 1 \\
1 & -1 & 1 & 1 & -1 \\
2 & 0 & 2 & -1 & 0 \\
3 & 1 & -1 & 0 & -1 \\
3 & -1 & -1 & 0 & 1
\end{tabular}

The top row merely names the conjugacy classes (by the familiar cycle structure for a permutation on four points). Below are five rows; each corresponds to an irreducible matrix representation. (Definition: a representation of a group $F$ to $G L(n, \mathbb{C})$ is irreducible if every subspace invariant under $F$ has dimension 0 or $n$.) For a given row, the entry below a conjugacy class gives the trace of a representing matrix. So, the dimension of the matrices is the first entry of each row. The trivial representation corresponds to the first row, the sign representation to the second, the action on 3-space by rotations on a cube to the fifth, and so on. For example, to exhibit the 5-dimensional complex representations of the group, one simply partitions 5 by integers 1,2 and 3 and lists all corresponding combinations of irreducibles. So, $5=1+1+1+1+1,1+1+1+2,1+1+3,1+2+2$, and $2+3$ which gives $6+4.1+3.2+2.1+1.2=20$ classes of homomorphisms from Sym $_{4}$ to $G L(5, \mathbb{C})$.

For homomorphisms into algebraic groups, there is an analogue of the finiteness of the number of representations of a given degree: if $G$ is an algebraic group and $F$ is a finite group, then $\operatorname{Hom}(F, G) / G$ is a finite set. Proofs of this nice result are given by Kulshammer [Kul], Prasad, Slodowy [Slo]; all depend on [Weil] which shows that the algebraic variety $\operatorname{Hom}(F, G) / G$ ) has dimension 0 .

The study of homomorphisms from a finite group to a general algebraic group is not as neat as the special case where the algebraic group is a general linear group. Recall that a general complex algebraic group $G$ has the following structure: the index of the connected component of the identity $G^{0}$ in $G$ is finite, and there is a maximal unipotent normal subgroup $R$ (the unipotent radical) such that $G^{0} / R$ is a central product of a torus and a finite number of quasisimple algebraic groups. (A group $H$ is a central product of subgroups $H_{1}, H_{2}, \ldots, H_{r}$ if $H=H_{1} H_{2} \ldots H_{r}$ and $\left[H_{i}, H_{j}\right]=1$ when $i \neq j$. We write $H=H_{1} \circ H_{2} \circ \ldots \circ H_{r}$. The definition allows 
the $H_{i}$ to be abelian, but in usual practice at most one of the factors is abelian. A nonidentity group is simple if its only normal subgroups are the identity and the group itself. A group is quasisimple if it is perfect (equal to its commutator subgroup) and its quotient by the center is simple.) We limit ourselves here to a study of homomorphisms from a finite group to quasisimple algebraic groups. This means (up to finite index and central extensions) the classical groups (special linear, orthogonal and symplectic groups) and the five exceptional groups $G_{2}(\mathbb{C})$, $F_{4}(\mathbb{C}), E_{6}(\mathbb{C}), E_{7}(\mathbb{C}), E_{8}(\mathbb{C})$.

A finite subgroup is contained in an essentially unique maximal compact subgroup of a complex algebraic group (such as the special unitary group in $S L(n, \mathbb{C})$ ); however, there seems to be little advantage to this viewpoint for the classification program, so we choose the convenience of complex groups.

If $G$ is a classical subgroup of $G L(n, \mathbb{C})$ associated to a nondegenerate bilinear form, meaning $O(n, \mathbb{C})$ or $S p(n, \mathbb{C})$, then we can tell if the image of an $n$-dimensional representation of $F$ lies in a $G L(n, \mathbb{C})$-conjugate of $G$ by knowing the character and the squaring map on conjugacy classes in $F$. An irreducible complex representation supports a unique (up to scalar multiple) nonzero hermitian form, but it need not leave invariant a nonzero bilinear form. Irreducibility implies that the space of invariant bilinear forms is at most one dimensional, and therefore, by considering the symmetric and alternating parts (which are invariant), we see that an invariant bilinear form is symmetric or alternating. The condition for an irreducible representation to have an invariant bilinear form is that the character be real valued, and the condition that it be symmetric, respectively alternating, is that the Frobenius-Schur indicator be 1 or -1 , respectively (the indicator of a character $\chi$ is $\frac{1}{|F|} \sum_{g \in F} \chi\left(g^{2}\right)$; it is 0 in the irreducible nonreal case; see, e.g., [Fe], [I]). For example, in the group $S_{y m}, 10$ elements square to the identity, 6 elements square to a permutation with shape $2^{2}$, and 8 elements square to a permutation with shape $1^{1} 3^{1}$ : we deduce that the indicator of either of the 3 -dimensional representations is $\frac{1}{24}(10.3-6.1+8.0)=1$. Accordingly, the 3 -dimensional irreducible representations of $S_{y m}$ afford homomorphisms to the orthogonal group. For a reducible character of degree $n$, the condition for mapping through $G$ is that the multiplicity of any nonreal irreducible must equal that of its dual (complex conjugate) and that all real irreducible characters which occur with odd multiplicity must be of the same type as $G$ (orthogonal or symplectic).

Homomorphisms into $S L$ and $S O$ involve more subtle issues. For example, in the first of the three dimensional representations of $S_{y m}$, involutions have trace 1 and therefore have eigenvalues of $-1,1$ and 1 , and a determinant of -1 . It follows that the image of this representation does not lie in a conjugate of $S O(3, \mathbb{C})$, whereas a similar calculation shows that the other three dimensional representation does lie in a conjugate of $S O(3, \mathbb{C})$. The following example shows that the character table alone does not determine embeddings into the algebraic group $S L$. For any prime $p$, there are two nonabelian groups of order $p^{3}$ and they have the same character table. The faithful characters of these groups all have degree $p$. For one of these groups, the image of any embedding in $G L(p, \mathbb{C})$ lies in $S L(p, \mathbb{C})$ and for the other it does not.

The conjugacy question, so easily resolved for $G L(n, \mathbb{C})$, can be complicated for general algebraic groups, and is indeed a serious matter for the classification of finite subgroups of exceptional groups. However, some of the nice features of maps 
from a finite group to $G L(n, \mathbb{C})$ are enjoyed elsewhere. Conjugacy of two such maps into the classical groups $O(n, \mathbb{C})$ and $S p(n, \mathbb{C})$ depends only on the character, i.e., on conjugacy in $G L(n, \mathbb{C})$. This is not trivial to prove; basic references are [Frob] [Mal][Tits55]; see Section 3 of [GrG2].

Some examples will indicate the difficulty with formulating a theory of conjugacy in other algebraic groups. There are infinitely many embeddings $\operatorname{PSL}(n, q) \rightarrow$ $P G L(m, \mathbb{C})$ which are elementwise conjugate but not conjugate [FoG]. A series of examples due to Borovik, Griess, and Larsen [Bor89] [Bor90][GrElAb] [Lar1] show that for exceptional groups $G$ other than $G_{2}(\mathbb{C})$, there are pairs of embeddings of finite groups $F$ in $G$ which are conjugate in $G L(V)$, for any faithful module $V$ for $G$, but which are not conjugate in $G$.

Conjugacy in exceptional algebraic groups. The problem of determining the conjugacy classes of projective embeddings of finite simple groups in exceptional Lie groups is not settled (see Table PE on page 84). Here are the general results known to us.

(1) For embeddings of finite groups in $G_{2}(\mathbb{C})$, one just checks the degree 7 character [Lar1][GrG2]. More is true: $G_{2}(\mathbb{C})$ strongly controls conjugacy of its subsets with respect to $G L(7, \mathbb{C})$; i.e. if subsets $A$ and $B$ of $G_{2}(\mathbb{C})$ and $g \in G L(7, \mathbb{C})$ satisfy $A^{g}=B$, there is a factorization $g=c h$, where $c \in C(A)$ and $h \in G_{2}(\mathbb{C})$ [GrG2]. A classification of finite subgroups of $G_{2}(\mathbb{C})$ is given in [GrG2], which gives shorter embedding proofs than in [CW83] and which solves the conjugacy problem. See Table PE and Section 5.

(2) Two subsets of $F_{4}(\mathbb{C})$ are conjugate if and only if they are conjugate in $E_{6}(\mathbb{C})$. A strong control of conjugacy result as in (1) applies to the containment of $F_{4}(\mathbb{C})$ in $E_{6}(\mathbb{C})[\mathrm{GrG} 2]$. This criterion is used in [F3] to settle conjugacy questions involved with embeddings of $A l t_{5}$ in $F_{4}(\mathbb{C})$ and $E_{6}(\mathbb{C})$.

\section{Classification of finite quasisimple subgroups}

Now we turn to the question of classifying finite subgroups. Usually one would not attempt to list all finite subgroups explicitly for a given algebraic group (though $S L(2, \mathbb{C})$ is an exception). For example, there are infinitely many finite subgroups of a maximal torus, and each of these may be extended upwards by subgroups of the Weyl group. In the classical algebraic groups (general linear, symplectic and orthogonal groups), finite subgroups are accounted for by character theory (see Section 1). From now on we therefore focus on finite subgroups of the exceptional groups. Our classification is mainly about such subgroups which are central extensions of the finite simple groups.

Definitions. An essential central extension of the finite group $F$ is an exact sequence of groups $1 \rightarrow Z \rightarrow K \rightarrow F \rightarrow 1$ such that $Z \leq Z(K) \cap K^{\prime}$. The group $Z$ is necessarily finite. If $Z$ is as large as possible, it is the Schur multiplier of $F$ (unique up to isomorphism) and $K$ is called a covering group of $F$.

A covering group of $F$ is unique up to isomorphism if $F$ is perfect (i.e., $F^{\prime}=F$ ), but not in general. Any essential central extension of $F$ is a quotient of a covering group of $F$. 
Definitions. A projective embedding of a finite group is a homomorphism from one of its covering groups whose kernel is in the center.

A quasisimple group $H$ is a quotient of the unique covering group of $H / Z(H)$. We point out that a projective embedding of a quasisimple group is just an embedding of a (possibly different) quasisimple group (both of these are central extensions of a common finite simple group).

Experience shows that the greater generality of studying projective embeddings of finite simple groups rather than just embeddings is done at little more cost and is actually quite useful. For example, a study of homomorphisms from the simple group of order 60 (called by the several names: the icosahedral group, Alt ${ }_{5}$, $P S L(2,4)$ and $P S L(2,5))$ to $E_{8}(\mathbb{C})$ requires an intermediate study of maps to a closed subgroup $H$ of type $D_{8}$. The group $H$ is isomorphic to the half spin group $H \operatorname{Spin}(16, \mathbb{C})$, and for calculations it is convenient to study its projective representation of dimension 16 since the smallest dimension of an ordinary nontrivial representation of $H$ is 128 . In such a projective representation of $H$, a subgroup isomorphic to $P S L(2,5)$ may correspond to a subgroup of $G L(16, \mathbb{C})$ which is isomorphic to $P S L(2,5)$ or $S L(2,5)$. The same is true for a study of embeddings of $S L(2,5)$ in $E_{8}(\mathbb{C})$. So, it is natural to study embeddings of $P S L(2,5)$ and $S L(2,5)$ together. Another example involves the simple group $P S L(2,37)$, which embeds in $E_{7}(\mathbb{C})$ (adjoint form) but not in $2 E_{7}(\mathbb{C})$ (simply connected form). For calculations with $E_{7}$, it is convenient to use the nontrivial module of smallest dimension, 56 , on which $2 E_{7}(\mathbb{C})$ acts faithfully. So, in practical terms, to study projective embeddings of the simple group $P S L(2,37)$ into a group of type $E_{7}$, one is really dealing with an embedding result for the quasisimple group $S L(2,37)$.

First, we indicate roughly what to expect from a classification of subgroups of an algebraic group. An old theorem of C. Jordan ((14.12), [I]) says that there exists a function $f(n)$ so that if $F$ is a finite subgroup of $G L(n, \mathbb{C})$, there exists a normal abelian subgroup $A$ of $F$ such that $|F: A| \leq f(n)$. A corollary is that $G L(n, \mathbb{C})$ contains only finitely many isomorphism types of finite quasisimple groups. The same is therefore true of any algebraic subgroup of $G L(n, \mathbb{C})$. However, the Jordan bound is not of practical use.

To classify finite quasisimple subgroups of an exceptional group $G$, the general strategy is to assume completeness of the list of finite simple groups and go through each family and limit possibilities. The possibilities not eliminated are then studied and proven to exist, by a variety of methods. Note that there is a chain of simply connected groups

$$
G_{2}(\mathbb{C})<F_{4}(\mathbb{C})<3 E_{6}(\mathbb{C})<2 E_{7}(\mathbb{C})<E_{8}(\mathbb{C})
$$

Existence of a projective embedding of a finite simple group in some exceptional algebraic group is equivalent to existence of projective embeddings in $E_{8}(\mathbb{C})$. The history in Section 5 treats the individual exceptional groups in greater detail.

Given a quasisimple algebraic group, one can use easy observations to eliminate most finite simple groups as possible subgroups. An elementary Lie algebra result that we use many times is the theorem of Borel-Serre [BS]. It says that a finite supersolvable group (defined below) acting on a finite dimensional complex Lie algebra stabilizes a Cartan subalgebra. Thus, its image in the automorphism group of this Lie algebra lies in the normalizer of a maximal torus. (Definition: a group is supersolvable if there is a finite length chain of normal subgroups in which the 
successive quotients are cyclic.) A finite solvable subgroup of an algebraic group need not normalize a torus, e.g., $A_{l} t_{4}$ in $P S L(2, \mathbb{C})$.

Here is a typical application of the Borel-Serre theorem to embeddings. Let $F \cong P S L(2, p)$, for an odd prime $p$, and assume that $F$ is contained in $G:=E_{8}(\mathbb{C})$. Write $B$ for the image modulo scalars of the subgroup of upper triangular matrices in $S L(2, p)$, that is,

$$
\left\{\left(\begin{array}{cc}
a^{-1} & b \\
0 & a
\end{array}\right) \mid a, b \in \mathbb{F}_{p}, a \neq 0\right\} \quad \bmod \text { scalars }
$$

and write $U$ for the normal subgroup of order $p\left(a=1, b \in \mathbb{F}_{p}\right)$. Then $B / U$ is cyclic of order $\frac{p-1}{2}$. Since $B$ is supersolvable it is contained in the normalizer $N$ of a torus $T$ of $E_{8}(\mathbb{C})$. The quotient $T / N$ is the Weyl group $W=W\left(E_{8}\right)$ (a group of order $2^{14} 3^{5} 5^{2} 7$ ). Since $T$ is abelian, the kernel of $B \rightarrow W$ is a normal abelian subgroup of $B$, so is 1 or $U$. The subgroup of diagonal matrices mod scalars therefore embeds in $W$, and this group is cyclic of order $\frac{p-1}{2}$. In the Weyl group, the order of an element is at most 30 (this follows by work with cyclotomic polynomials since $W$ has a faithful eight dimensional rational representation). We conclude that $\frac{p-1}{2} \leq 30$, whence $p \leq 61$. (In fact, there is an embedding of $P S L(2,61)$ in $E_{8}(\mathbb{C})$ !)

Another application of $[\mathrm{BS}]$ is a quick proof that many sporadic simple groups do not embed in $E_{8}(\mathbb{C})$. The Mathieu group $M_{23}$ of order $2^{7} 3^{2} 5.7 .11 .23$ [Gor] [GrTwelve] has a Sylow 23-normalizer of the form 23: 11, a nonabelian supersolvable group. Since 11 does not divide the order of the Weyl group $W\left(E_{8}\right)$, the Borel-Serre theorem implies that this Sylow normalizer does not embed in $E_{8}(\mathbb{C})$, whence neither does $M_{23}$. Any sporadic group (or covering) which contains $M_{23}$ is also eliminated from consideration as a subgroup of $E_{8}(\mathbb{C})$ (this applies to nine other sporadics). Using these and other techniques, one can show that the quasisimple groups of sporadic type which embed in $E_{8}(\mathbb{C})$ are just $M_{11}, 2 \cdot M_{12}$ and $2 \cdot H J$.

When $q$ is a prime power, we would like restrictions on values $n, q$ for which there is an embedding of $\operatorname{PSL}(n, q)$ in $E_{8}(\mathbb{C})$. When $n=2$ and $q$ is not prime, the Borel-Serre theorem may not be used since the subgroup of upper triangular matrices in $\operatorname{PSL}(2, q)$ is not supersolvable (though it is solvable). Results about elementary abelian $p$-subgroups and their normalizers in algebraic groups do give restrictions. For example, an elementary abelian $p$-subgroup of $E_{8}(\mathbb{C})$ has rank at most 8 if $p$ is odd and at most 9 if $p=2[\mathrm{CS}][\mathrm{GrElAb}]$. A corollary is that if $P S L\left(3, p^{k}\right)$ embeds in $E_{8}(\mathbb{C})$, then $k \leq 4$, due to a rank $2 k$ subgroup

$$
\left\{\left(\begin{array}{ccc}
1 & 0 & a \\
0 & 1 & b \\
0 & 0 & 1
\end{array}\right) \mid a, b \in \mathbb{F}_{p^{k}}\right\} \quad \bmod \text { scalars. }
$$

Using more detailed results, one can get constraints for all $k$ on Borel subgroups of $P S L\left(2, p^{k}\right)$ which embed in $E_{8}(\mathbb{C})$. If the Borel subgroup is in a torus normalizer, we get $\frac{p^{k}-1}{2} \leq 30$. If not, the normal subgroup $U$ is nontoral, in which case it is contained in a maximal nontoral elementary abelian $p$-group from a finite list [GrElAb], and we therefore get other bounds on $p^{k}$. For example, if $k \geq 3$ and $U$ is nontoral, the tables in [GrElAb] show immediately that $p \leq 5$. When $p=5, N(U)$ 
is isomorphic to the semidirect product $5^{3}: S L(3,5)$ (a subgroup of the affine group $A G L(3,5))$. Since $P S L(2,125)$ contains an element of order $\frac{5^{3}-1}{2}=62$ and $N(U)$ does not, we have a contradiction to existence of an embedding of $P S L(2,125)$. The analogous discussion for $p=3$ leads to $N(U) \cong 3^{3}: S L(3,3)$, which does contain an element of order $\frac{3^{3}-1}{2}=13$, no contradiction. Indeed, there is a subgroup $P S L(2,27)$ in $F_{4}(\mathbb{C})$. The case $p=2$ is realized by a subgroup $P S L(2,8)$ in $G_{2}(\mathbb{C})$.

In general, one combines "local arguments" (studies of nonidentity $p$-subgroups and their normalizers) with character theory to eliminate or limit the cases which require detailed study. Suppose that we consider a finite quasisimple group $F$ and its possible embeddings in $G:=E_{8}(\mathbb{C})$. We may have tried to obtain a "local contradiction" as above but failed. This situation usually arises when $F$ is not too large, so it is then reasonable to study fusion patterns, that is the assignments of classes of elements of order $n$ in $F$ to classes of order $n$ in $G$. If $n \leq 8$, such classes are fully listed $[\mathrm{CG}][\mathrm{FrG}]$. So, we can ask if there is a character of $F$ which is the restriction of the adjoint character of $G$ to a putative subgroup $F$ (this is where all possible fusion patterns need to be considered). Actually, we may restrict ourselves to work with partial characters (supported at just some conjugacy classes, of elements of orders at most 8), but this can be enough to get a contradiction. A nice example is the case of $S z(32)$. If $S z(32)$ were embeddable in $E_{8}(\mathbb{C})$, the restriction of the degree 248 adjoint representation to $S z(32)$ would be a sum of two degree 124 irreducibles. Moreover, the 248-dimensional module $M$ affording this character must support a Lie algebra structure invariant under the action of $S z(32)$. The Lie product provides an invariant homomorphism in $H_{o m}(32)(M \wedge M, M)$ whose image is all of $M$. However, the irreducible characters of degree 124 for $S z(32)$ do not appear in the exterior square of their sum, whence there is no nontrivial invariant Lie algebra structure, a contradiction.

Character theory was critical in our construction of embeddings into $E_{8}(\mathbb{C})$ of $S z(8)$, a finite simple group of order $29120=2^{6} 5.7 .13$. We first studied traces of elements of orders $2,4,5$ to deduce that the restriction of the degree 248 adjoint character is $14_{a}+14_{b}+64+65_{x}+91$ where $65_{x}$ denotes one of three irreducibles in an orbit under $\operatorname{Out}(S z(8)) \cong 3$. It helps to start with the observations that in $L$, an element of order 5 is rational (so can be in only two of the fourteen $E_{8}(\mathbb{C})$ classes of elements of order $5[\mathrm{CG}]$ ) and that if $U$ is a Sylow 2-group, the center is an elementary abelian group of order 8 with the property that every nonidentity element is in a single orbit under the action of $N(U)$. There are two such "pure" elementary abelian eights-groups in $E_{8}(\mathbb{C})$ up to conjugacy, one of which is $2 A$ pure and one $2 B$-pure $[\mathrm{CG}]$. (Pure means that all nonidentity elements of the subgroup lie in a single conjugacy class; the notations $2 A, 2 B$ refer to the two $E_{8}(\mathbb{C})$ conjugacy classes of elements of order 2.) The $2 A$-pure eights-group has centralizer in $E_{8}(\mathbb{C})$ of the form $2^{3} \times F_{4}(\mathbb{C})$. Since $Z(U) \leq U^{\prime}, Z(U)$ is not $2 A$-pure. So, the involutions of $Z(U)$ are of $2 B$-type. Forcing the above character decomposition is now an exercise by hand with the character table of $S z(8)$ and the list of elements of small order in $E_{8}(\mathbb{C})[C G]$. As a second check, a computer was used to survey partial characters supported only at the elements of orders 1, 2 and 4 in $S z(8)$, and the same conclusion was reached. For a few other finite quasisimple groups, it was important to know the relevant 248-dimensional characters for classifying embeddings. 
For many of the hard cases, conjugacy of embeddings was settled at the same time as existence of embeddings. These cases are about situations which are sufficiently rigid to permit just a few possibilities (such as $P S L(2, q)$ in $E_{8}(\mathbb{C})$, for certain large $q$, and $P S U(3,8)$ in $E_{7}(\mathbb{C})$ ). At the other extreme are small $L$ with many embeddings. See Table PE on page 84 for a summary of information about $A_{l} t_{5}$ and $S L(2,5)$, due to Darrin Frey [F1][F2]; and $P S L(2,7)$ and $S L(2,7)$, due to Mike Kantor $[\mathrm{K}]$. These are the only classification results known for finite simple groups with large numbers of embedding classes (say, more than five). For all four of these finite groups, a fusion analysis and restriction of the adjoint character were considered. The resulting class function must be a nonnegative integer linear combination of characters, if there is an associated embedding. Many fusion patterns which passed this initial test were rejected later by local analysis in $E_{8}(\mathbb{C})$; i.e., the embedding of the local subgroups of such a group in $E_{8}(\mathbb{C})$ led to particular group theoretic conditions on Lie subgroups which did not hold. For instance, existence of an embedding might imply that a coset of a subgroup of $E_{8}(\mathbb{C})$ must contain elements of order 2 and this condition could be disproved by group theoretic examination. In principle, a given fusion pattern (which determines a degree 248 character) could be associated to a large finite number of embeddings. However, this number is 1 for nearly every fusion pattern for these four groups. It is known to be 2 in a few cases, and there remain a few unsettled cases.

\section{TABle of PRojective EMBEDdings of Finite Simple GRoups INTO EXCEPTIONAL ALGEBRAIC GROUPS}

We next give a table which summarizes the state of the classification. For groups $F$ and $G, \operatorname{Emb}(F, G)$ and $\operatorname{Proj} \operatorname{Emb}(G)$ denote the set of embeddings and projective embeddings respectively. The quotient by $G$ of one of these denotes its equivalence classes by $G$-conjugacy. Other notation is compatible with that of [CG], [GrElAb], which is derived from standard notation in finite group theory and the theory of Chevalley groups [Gor][Hup][Car].

A finite simple group $L$ appears in column 2 of Table PE if and only if it has a projective embedding in $E_{8}(\mathbb{C})$. If so, column 1 lists the isomorphism type $z$ of the center of a quasisimple group $S$ whose central quotient is $L$. For all such $z$, we give what we know about embeddings in exceptional groups in columns 3,4 , $5,6,7$; for at least one $z$, there is an embedding. That is, we try to specify which central extensions of finite simple groups occur in the exceptional groups. Since $E_{8}(\mathbb{C})$ contains all exceptional groups in simply connected versions via a chain in the order $G_{2}(\mathbb{C})<F_{4}(\mathbb{C})<3 E_{6}(\mathbb{C})<2 E_{7}(\mathbb{C})<E_{8}(\mathbb{C})$, a projective embedding into one of them is a projective embedding into all overgroups. Note that the columns of Table PE are headed by simply connected versions of the exceptional groups.

A numerical table entry $m$ (or $\geq m$ ) means the quasisimple finite group $S$ in that row has $m$ (or at least $m$ ) equivalence classes of embeddings in the algebraic group $H$ of that column, i.e., $m=|E m b(S, H) / H|$. A numerical entry in parentheses $(n)$ refers to the number $n=|\operatorname{Emb}(S, H) / \operatorname{Aut}(S) \times H|$. An entry $y$ ("yes") means $\geq 1$, an entry ? means that we do not know whether there are any embeddings, and an entry $Z$ or $S$ means that there are unsettled cases only for zero dimensional centralizer or small dimensional centralizer, respectively. 
TABLE PE. The finite simple groups with a projective embedding in $E_{8}(\mathbb{C})$

\begin{tabular}{|c|c|c|c|c|c|c|c|}
\hline$z$ & $\begin{array}{l}\text { Finite } \\
\text { Simple } \\
\text { Group }\end{array}$ & $G_{2}(\mathbb{C})$ & $F_{4}(\mathbb{C})$ & $3 E_{6}(\mathbb{C})$ & $2 E_{7}(\mathbb{C})$ & $E_{8}(\mathbb{C})$ & $\begin{array}{l}\text { Reference, } \\
\text { Comments }\end{array}$ \\
\hline 1 & Alt $_{5}$ & 4 & $13(8)$ & $15(10)$ & $y$ & $\geq 31(\geq 19) Z$ & {$[\mathrm{~F} 1,2,3][\mathrm{GRQ}]$} \\
\hline 2 & & 4 & $12(21)$ & $18(32)$ & $y$ & $\geq 103(\geq 58) S$ & {$[\mathrm{~F} 1,2,3][\mathrm{GRQ}]$} \\
\hline 1 & $A l t_{6}$ & 0 & $3 A_{2} A_{2}$ & $y$ & $y$ & $y$ & {$[\mathrm{CG}][\mathrm{GrG} 2]$} \\
\hline 2 & & 0 & $4 D_{3}$ & $y$ & $y$ & $y$ & {$[\mathrm{CG}][\mathrm{GrG} 2]$} \\
\hline 3 & & $2(1) ; 3 A_{2}$ & $3 A_{2}$ & $y$ & $y$ & $y$ & {$[\mathrm{CG}][\mathrm{GrG} 2][\mathrm{GRQ}]$} \\
\hline 6 & & 0 & 0 & $6 A_{5}$ & $y$ & $y$ & {$[\mathrm{CG}][\mathrm{GrG} 2][\mathrm{GRQ}]$} \\
\hline 1 & $\mathrm{Alt}_{7}$ & 0 & 0 & $6 A_{5}$ & $2^{2} D_{6}$ & $y$ & {$[\mathrm{CG}]$} \\
\hline 2 & & 0 & $4 D_{3}$ & $y$ & $y$ & $y$ & {$[\mathrm{CG}]$} \\
\hline 3 & & 0 & $0^{\circ}$ & $6 A_{5}$ & $y$ & $y$ & {$[\mathrm{CG}][\mathrm{GRQ}]$} \\
\hline 6 & & 0 & 0 & $6 A_{5}$ & $y$ & $y$ & [CG] [GRQ] \\
\hline 1 & $\begin{array}{l}\operatorname{Alt}_{n} \\
n=8\end{array}$ & & & & & & \\
\hline $\begin{array}{l}1 \\
2\end{array}$ & $\begin{array}{l}n=8, \ldots, 10 \\
n=8, \ldots, 17\end{array}$ & $\begin{array}{l}0 \\
0\end{array}$ & ${ }^{0}$ & $n<11$ & $\begin{array}{c}y: n=8 \\
n<13\end{array}$ & $n \leq 10 ; 3 A_{8}$ & {$[\mathrm{CG}]$} \\
\hline & $\operatorname{PSL}(2,4) \rightarrow$ & 0 & $n=9$ & $n=11$ & & $n \leq 17 ; 2 D_{8}$ & $\begin{array}{l}{[C G][C W 95]} \\
\quad \cong \text { Alt }_{5}\end{array}$ \\
\hline & $\operatorname{PSL}(2,5) \rightarrow$ & & & & & & $\cong A l t_{5}$ \\
\hline 1 & $P S L(2,7)$ & 2 & $y$ & $y$ & $y$ & $\geq 33(\geq 16) S$ & {$[\mathrm{~K}][\mathrm{GRQ}]$} \\
\hline 2 & & 0 & $2 \mathrm{~B}_{4}$ & $y$ & $y$ & $\sum 55(\geq 22) S$ & {$[\mathrm{~K}]$} \\
\hline 1 & $\begin{array}{l}P S L(2,8) \\
\operatorname{PSL}(2,9) \rightarrow\end{array}$ & $3(1) P$ & $y$ & $y$ & $y$ & $y$ & $\begin{array}{l}{[\mathrm{CW} 83][\mathrm{GrG} 2]} \\
\simeq \mathrm{Alt}_{6}\end{array}$ \\
\hline 1 & $\operatorname{PSL}(2,11)$ & 0 & 0 & $5 A_{4}, 2 D_{5}$ & $y$ & $y$ & {$[\mathrm{CG}][\overline{\mathrm{C}} \mathrm{W} 83][\mathrm{GRQ}]$} \\
\hline 2 & & 0 & 0 & $2 D_{5}$ & $y$ & $y$ & [CG] [CW83][GRQ] \\
\hline 1 & $P S L(2,13)$ & 2(1) $P$ & $y$ & $\geq 6(\geq 3)$ & $y$ & $y$ & [CW83] [CW93][GrG2] \\
\hline 2 & & 0 & 0 & $2(\overline{1}) ; 2 \bar{A}_{1} A_{5}$ & $y$ & $y$ & [GRQ] \\
\hline 1 & $P S L(2,16)$ & 0 & 0 & $\begin{array}{c}0 \\
0\end{array}$ & $\begin{array}{l}g \\
0\end{array}$ & $2 D_{8}$ & {$[\mathrm{CG}][\mathrm{CW} 95][\mathrm{GRQ}]$} \\
\hline 1 & $P S L(2,17)$ & 0 & $2(1) ; 2 B_{4}$ & $y$ & $4 A_{7}$ & $3 A_{8}^{8}$ & {$[\mathrm{CG}]$} \\
\hline 2 & & 0 & 0 & 0 & 0 & $2 D_{8}$ & {$[\mathrm{CG}]$} \\
\hline 1 & $P S L(2,19)$ & 0 & 0 & $\geq 4(1) P$ & $y$ & $A_{8}$ & [CG][CW97][GRQ] \\
\hline 2 & & 0 & 0 & 0 & $y$ & $y$ & {$[\mathrm{~S} 96] ; y: P G L(2,19)$} \\
\hline 1 & $P S L(2,25)$ & 0 & $P$ & $F_{4}$ & $y$ & $y$ & [CW97] \\
\hline 2 & & 0 & 0 & 0 & ? & $2 D_{8}$ & {$[\mathrm{CG}]$} \\
\hline 1 & $P S L(2,27)$ & 0 & $\geq 3(1) P$ & $y$ & $y$ & $y$ & [CW97] \\
\hline 2 & & 0 & 0 & $\begin{array}{l}9 \\
0\end{array}$ & $\begin{array}{l}\text { ? } \\
?\end{array}$ & $\begin{array}{l}9 \\
?\end{array}$ & [CW97] \\
\hline 1 & $P S L(2,29)$ & 0 & 0 & 0 & 0 & $2 B_{7} \leq 2 D_{8}$ & {$[\mathrm{CG}], \mathrm{p} .370[\mathrm{GRQ}]$} \\
\hline 2 & & 0 & 0 & 0 & $y P$ & 20 & [SP] [GRQ] \\
\hline 1 & $P S L(2,31)$ & 0 & 0 & 0 & 0 & $\geq 3(2) P$ & $\begin{array}{l}\text { [S96] [GR31]; } \\
3(2) \text { for } \operatorname{PGL}(2,31)\end{array}$ \\
\hline 1 & $P S L(2,32)$ & 0 & 0 & 0 & 0 & 5(1) $P$ & [GR31] \\
\hline 2 & $P S L(2,37)$ & 0 & 0 & 0 & 2(1) $P$ & $2(1)$ & {$[\mathrm{KR}][\mathrm{CG}],(5.2 .10)$} \\
\hline 1 & $P S L(2,41)$ & 0 & 0 & 0 & 0 & $3(1) P$ & [GR41] \\
\hline 1 & $\operatorname{PSL}(2,49)$ & 0 & 0 & 0 & 0 & 2(1) $P$ & [GR41] \\
\hline 1 & $\begin{array}{l}\operatorname{PSL}(2,61) \\
\operatorname{PSL}(3,2) \rightarrow\end{array}$ & 0 & 0 & 0 & 0 & 2(1)P & $\begin{array}{l}{[\mathrm{CGL}][\mathrm{GRQ}]} \\
\simeq \operatorname{PSL}(2,7)\end{array}$ \\
\hline 1 & $\operatorname{PSL}(3,3)$ & 0 & $y P$ & $y$ & $y$ & $y$ & in $3^{3}: S L(3,3) ;[\mathrm{Alek}][\mathrm{CG}][\mathrm{GrElAb}]$ \\
\hline 2 & $\operatorname{PSL}(3,4)$ & 0 & 0 & 0 & $4 A_{7}$ & $y$ & 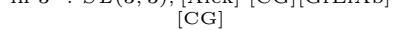 \\
\hline 4 & & 0 & 0 & 0 & 0 & $8 A_{7}$ & {$[\mathrm{CG}][\mathrm{GRQ}]$} \\
\hline 6 & & 0 & 0 & $6 A_{5}$ & $y$ & $y$ & {$[\mathrm{CG}]$} \\
\hline 1 & $\begin{array}{l}\operatorname{PSL}(3,5) \\
\operatorname{PSL}(4,2) \rightarrow\end{array}$ & 0 & 0 & 0 & 0 & $y P$ & in $5^{3}: S L(3,5) ;[$ Alek $][\mathrm{CG}][\mathrm{GrElAb}]$ \\
\hline 1 & $P S U(3,3)$ & 1 & $y$ & $y$ & $y$ & $y$ & {$[\mathrm{CW} 83][\mathrm{GrG} 2]$} \\
\hline 1 & $P S U(3,8)$ & 0 & 0 & 0 & $1 P$ & 1 & {$[\mathrm{GRU}][\mathrm{GRQ}]$} \\
\hline 1 & $P S U(4,2)$ & 0 & 0 & $6 A_{5}$ & $y$ & $y$ & $\cong \Omega^{-}(6,2) \cong W_{E_{6}}^{\prime}[\mathrm{CG}][\mathrm{GRQ}]$ \\
\hline 2 & & 0 & $4 D_{3}$ & $y$ & $y$ & $2 D_{8}$ & \\
\hline 6 & $P S U(4,3)$ & 0 & 0 & $6 A_{5}$ & $y$ & $y$ & {$[\mathrm{CG}]$} \\
\hline 1 & $\mathrm{PSO}^{+}(8,2)$ & 0 & ? & $?$ & ? & $2^{2} D_{4} D_{4}$ & [CG] \\
\hline 2 & $\mathrm{PSO}^{+}(8,2)$ & 0 & $?$ & ? & ? & $2^{2} \mathrm{D}_{4} \mathrm{D}_{4}$ & [CG] \\
\hline $2^{2}$ & $\mathrm{PSO}^{+}(8,2)$ & 0 & $2^{2} D_{4}$ & $y$ & $y$ & $2^{2} \mathrm{D}_{4} \mathrm{D}_{4}$ & [CG] \\
\hline 2 & $\begin{array}{l}\operatorname{PSp}(4,3) \rightarrow \\
\quad \operatorname{PSp}(4,5)\end{array}$ & 0 & 0 & P & 0 & R & $\cong P S U(4,2)$ \\
\hline 1 & $\operatorname{PSp}(6,2)$ & $\begin{array}{l}0 \\
0\end{array}$ & 0 & $\begin{array}{l}0 \\
0\end{array}$ & $\begin{array}{l}0 \\
?\end{array}$ & $2^{B_{6}}{ }^{2}$ & $\begin{array}{c}\text { [CG]; Sec. } 6[\mathrm{GRQ}] \\
\simeq \Omega(7,2) \cong W_{F^{\prime}}^{\prime}[\mathrm{GRQ}] ;[\mathrm{CG}]\end{array}$ \\
\hline 2 & & 0 & ${ }_{2}^{2} \mathrm{D}_{4}$ & $y$ & $y$ & $2^{2} D_{4}^{2}$ & {$[\mathrm{CG}]$} \\
\hline 1 & $S z(8)$ & 0 & 0 & 0 & 0 & $3(1) \stackrel{4}{P}$ & Sec. $6[$ GR 8$]$ \\
\hline & $G_{2}(2)^{\prime} \rightarrow$ & & & & & & $\cong P S U(3,3)$ \\
\hline 1 & $G_{2}(3)$ & 0 & 0 & 0 & 0 & $D_{7}$ & [CG][GRQ] \\
\hline 1 & ${ }^{3} \mathrm{D}_{4}(2)$ & 0 & $y P$ & $y$ & $y$ & $y$ & [CW97] \\
\hline 1 & ${ }^{2} F_{4}(2)^{\prime}$ & 0 & 0 & $y P$ & $y$ & $y$ & [CW97]; $y:{ }^{2} F_{4}(2)$ \\
\hline 2 & $H J$ & 0 & 0 & $6 A_{5}$ & $y$ & $y$ & {$[\mathrm{CG}]$; see Sec. 4} \\
\hline 1 & $M_{11}$ & 0 & 0 & $D_{5}$ & $y$ & $y$ & {$[\mathrm{CG}]$} \\
\hline 2 & $M_{12}$ & 0 & 0 & 0 & $2 B_{5} \leq 4 D_{6}$ & $y$ & {$[\mathrm{CG}]$; see Sec. $4[\mathrm{GRQ}]$} \\
\hline
\end{tabular}


A finite subgroup $F$ of a connected algebraic group $G$ with finite center is Lie primitive if and only if whenever $H$ is a Zariski closed subgroup of $G$ with $F \leq$ $H \leq G$, then $H$ is finite or $H=G$. If $F$ is Lie imprimitive and an $H<G$ as above can be chosen so that $F \leq H^{0}$, we say that $F$ is intrinsically Lie imprimitive (via $H)$. An example: $E_{8}(\mathbb{C})$ contains a finite subgroup $F$ of the form $2^{1+3} \cdot G L(3,2)$, which is contained in a subgroup $H$ (the normalizer of the normal subgroup of order $2^{4}$ ) of the form $2^{4} . A_{1}^{8}(\mathbb{C}) \cdot 2^{3} \cdot G L(3,2)$ and in another subgroup $C$ of the form $2 E_{7}(\mathbb{C}) A_{1}(\mathbb{C})$ (the centralizer of the center of $F$ ). Since $C$ is connected, $F$ is intrinsically Lie imprimitive, but $F$ does not lie in $H^{0}$. For arbitrary connected $G$, we say that $F$ is Lie primitive in $G$ iff $F Z(G) / Z(G)$ is Lie primitive in $G / Z(G)$.

In the table, an entry $P$ means all indicated cases are Lie primitive embeddings in that column's exceptional group. An entry giving an intermediate semisimple Lie group indicates existence of some (non Lie primitive) embedding via such a subgroup; in this case, there is no implication that all such embeddings occur this way.

The column marked "Reference, Comments" cites sources for information in a given row. An arrow means that an exceptional isomorphism is noted to the right. We do not describe finite overgroups of finite quasisimple Lie primitive subgroups. Normalizers are discussed in [CW97].

\section{Sketch OF The COMPUTER WORK ON HARD EMBEDDings}

All computer proofs of embeddings $L \leq G=E_{8}(\mathbb{C})$ have proceeded by building an embedding of the finite group $L$ into a Chevalley group of type $E_{8}$ over a field $F$ of positive characteristic prime to $|L|$ and applying the following Lifting Lemma [CGL] $[\mathrm{GrElAb}]$ to obtain an embedding in characteristic 0.

Lifting Lemma. Let $p$ be a prime number and $L$ be a finite group of order prime to $p$, such that $L$ embeds in a Chevalley group of type $E_{8}$ in characteristic $p$. Then, $L$ embeds in $E_{8}(\mathbb{C})$.

The computer constructions rely on being able to compute in a finite Chevalley group of type $E_{8}$ and in its 248-dimensional matrix representation. Thus, for the purposes of computer constructions, a Chevalley group of type $E_{8}(F)$ will always be considered as a subgroup of the matrix group $G L(248, F)$.

There are two fundamentally different computational strategies that have been employed. In the first strategy, we begin with an explicit matrix representation of the group $E_{8}(F) \leq G L(248, F)$, and working within the matrix group $E_{8}(F)$, we build up a subgroup isomorphic to $L$. In the second strategy, we start instead with an explicit matrix representation $L \leq G L(248, F)$, and we build a copy of $E_{8}(F)$ between $L$ and $G L(248, F)$.

The first strategy tends to be successful in cases where the group $L$ is generated by a pair of subgroups $L_{1}$ and $L_{2}$ with a large intersection. This is because the construction works by building a copy $L_{1}^{*}$ of $L_{1}$ inside $E_{8}(F)$, then looking for a copy $L_{2}^{*}$ of $L_{2}$ by identifying a candidate $J$ for $L_{1}^{*} \cap L_{2}^{*}$ inside $L_{1}^{*}$ and trying to extend this candidate to a group $L_{2}^{*}$. There are usually several different choices for 
such an extension of $J$ to $L_{2}^{*}$, and most of these choices will prove fruitless. For each possible candidate for $L_{2}^{*}$ we must test whether $\left\langle L_{1}^{*}, L_{2}^{*}\right\rangle$ is a copy of $L$. If the centralizer in $E_{8}(F)$ of $J$ is large compared to the centralizer of $L_{2}$, there will be a great many ways to extend $J$ to a copy of $L_{2}$ and the method will be unwieldy.

For example, in our computer construction of embeddings of $L=P G L(2,31)$ [GR31], we view the group $L$ as generated by elements $s, t, u$ of respective orders 31 , 30, 2: the images of the $G L(2,31)$ elements

$$
\left(\begin{array}{ll}
1 & 1 \\
0 & 1
\end{array}\right),\left(\begin{array}{ll}
1 & 0 \\
0 & 3
\end{array}\right),\left(\begin{array}{cc}
0 & 1 \\
-1 & 0
\end{array}\right)
$$

Two particularly useful subgroups of $L$ are: $\langle s, t\rangle \cong 31: 30$ and $\langle t, u\rangle \cong 30: 2$. We also remark that the element us has order 3 .

To build up a copy of $L$ in a Chevalley group of type $E_{8}$, we examine subgroups of $E_{8}(p)$ with structures $L_{1}^{*} \cong 31: 30$ and $L_{2}^{*} \cong 30: 2$ that intersect in a cyclic subgroup of order 30 . We pick a characteristic that will make it easy for us to locate elements of orders 30 and 31 in $E_{8}(p)$. Specifically, we choose characteristic $p=2791=3 \times 30 \times 31+1$, and we work with a particular copy of $E_{8}(p)$ inside $G L(248, p)$. We begin by selecting a copy of $L_{1}$, which we know can be found inside the normalizer of a split torus (by our choice of characteristic, and by preliminary consideration of characters of degree 248 of $L$ which could result from an embedding of $L$ in $\left.E_{8}(p)\right)$. We write $s$ for a generator of the cyclic Sylow 31-subgroup of $L_{1}$ and $t$ for a normalizing element of order 30 that has the property that $s^{t}=s^{3}$.

Again, preliminary consideration of $E_{8}$-feasible characters of $L$ shows that the centralizer of $t$ is just another split torus, $X$ say, of $E_{8}(p)$. Suppose now that $u_{0}$ inverts the torus $X$ under conjugation. The coset $X u_{0}$ gives the complete set of involutions in $E_{8}(p)$ that invert $t$. Our task is to examine all of these involutions and detect which (if any) together with $s$ and $t$ generate a copy of $L$.

We remark that it is a routine matter to obtain explicit matrices giving generators for $X$ and for the elements $s, t$, and $u_{0}$ that we have described. However, the group $X$ has $2790^{8}$ elements, and therefore, it is computationally infeasible to search all elements of the coset $X u_{0}$ for generators of $L$.

Recall that we are looking for an involution $u=x u_{0} \in X u_{0}$ with that additional property that $1=(u s)^{3}$. Rearrangement of this relation shows that: $x u_{0} s x u_{0} s=$ $s^{-1} x u_{0}$. Now let $h$ be an element of the Cartan subalgebra of the Lie algebra of $G$ that is fixed by $X$. In our computation $h$ is just represented as a $1 \times 248$ row vector. Then, because $h x=h$, we need an element $x$ that satisfies:

$$
h u_{0} s x u_{0} s=h s^{-1} x u_{0}
$$

This is an equality between two 248-dimensional vectors. Moreover, in (*), every quantity except $x$ is represented by a known vector or matrix. Hence, we can view $\left(^{*}\right)$ as 248 conditions on the unknown $x$. By using 8 basis vectors of the Cartan subalgebra fixed by $X$, we obtain $8 \times 248$ such conditions. These conditions are linear on the additive subspace of matrices spanned by the torus $X$. However, the torus $X$ is conjugate to a set of diagonal matrices, and therefore the additive space that it spans is also conjugate to a set of diagonal matrices, and thus has dimension at most 248 . 
We have $248 \times 8$ linear conditions on an unknown matrix $x$ in a 248-dimensional space. A simple Gaussian elimination produces the solution space: it turns out to be 3-dimensional. We can search this "small" space. The search produces exactly 3 candidates for embeddings of $L$ into $E_{8}(2791)$ (in [GR31] we show further that these are the only candidates over any extension field of $\left.\mathbb{F}_{2791}\right)$. We must still check that the three candidates really give copies of $L$ : in [GR31] this is accomplished by verifying a presentation for $L$.

We now outline an example of the second computational strategy for establishing Lie primitive subgroups of $E_{8}(\mathbb{C})$. In our construction [GR8] of an embedding of $L=S z(8)$, we begin with an explicit 248-dimensional representation $L \leq G L(248, F)$. Preliminary character theoretic calculations show that if $F$ contains 4 th and 7 th roots of unity and there exists an intermediate Chevalley group of type $E_{8}$, then the representation must break up as a sum of five distinct irreducible constituents with degrees $14,14,64,65$ and 91 . (There are three different possibilities for the 65-dimensional constituent, but the other constituents are uniquely determined). It is computationally preferable to work over a prime field, and it is desirable to work with the above roots of unity, so we choose to compute over the field $\mathbb{F}_{29}$, and with an appropriate representation of $L$ on a 248-dimensional space $V$ over $F$. We write $V_{14 a}, V_{14 b}, V_{64}, V_{65}$ and $V_{91}$ for the explicitly known irreducible constituents of the $L$-module $V$.

Rather than search for an intermediate subgroup, we search for an $L$-invariant Lie algebra of type $E_{8}$ on $V$. The existence of such a Lie algebra would give us an embedding of $L$ in the automorphism group of the algebra, a Chevalley group of type $E_{8}$.

We can view a Lie algebra structure on $V$ as a bilinear product $V \times V \rightarrow V$. Thus we seek a particular Lie element from a space parametrized by $\operatorname{Hom}_{L}(V \otimes V, V)$. Unfortunately this parameter space is 516-dimensional, so we cannot search it directly. However, we can apply the theory of modular Lie algebras to show that any $L$-invariant Lie product on $V$ must be determined by its effect on $V \times V_{14 a}$. In other words we need only search the smaller (27-dimensional) parameter space $H_{o m}\left(V \otimes V_{14 a}, V\right)$. This space is still too large to search directly; however, we can use the knowledge that $H_{o m}\left(V_{14 a} \otimes V_{14 a}, V\right)$ is just one dimensional to deduce further linear conditions that must be satisfied by a Lie element of $H_{o m}\left(V \otimes V_{14 a}, V\right)$. For example, the Jacobi identity at a triple of fixed vectors of $V_{14 a}$ leads to 248 linear conditions on $\operatorname{Hom}_{L}\left(V \otimes V_{14 a}, V\right)$ that must be satisfied by any Lie element. Further computations, with other consequences of the Jacobi identity that lead to linear conditions $\operatorname{Hom}_{L}\left(V \otimes V_{14 a}, V\right)$ and its tensor square, show that there is an essentially unique $L$-invariant Lie algebra of type $E_{8}$ on $V$. As a corollary we deduce that $S z(8)$ does embed in $E_{8}(\mathbb{C})$.

The second strategy, which we have just illustrated, tends to be successful when the adjoint module for $E_{8}(F)$ has relatively few irreducible constituents when viewed as an $L$-module. This is because, in order to construct $E_{8}(F)$, we construct an $L$-invariant Lie algebra on the natural 248-dimensional $L$-module. This is accomplished by searching the space of all $L$-invariant alternating products on the module. If the module has a large number of irreducible constituents, there will be an impossibly large number of $L$-invariant products to consider. This is the case where $L$ is $P S L(2,5), S L(2,5), P S L(2,7)$ or $S L(2,7)$. 


\section{HISTORY OF CLASSIFICATION WORK FOR EXCEPTIONAL GROUPS}

The earliest work on finite subgroups of exceptional groups was that of Cohen and Wales $[\mathrm{CW} 83]$ on $G_{2}(\mathbb{C})$. In 1984, Cohen and Griess began work on finite quasisimple subgroups of $E_{8}(\mathbb{C})$ and limited the possibilities $[\mathrm{CG}]$. There were quite a few unsettled cases. The analogous problem for $F_{4}(\mathbb{C})$ and $E_{6}(\mathbb{C})$ was considered by Cohen and Wales in the mid 80s [CW97]. In all these cases, one searches through a list of candidate simple groups for projectively embeddable ones. In the case of $G_{2}(\mathbb{C})$, Wales' work on finite linear groups of degree $7[\mathrm{~W}]$ gives a short list to consider. For the larger exceptionals, no straight classifications of linear groups of degrees $26,27,52,56,78,133$ or 248 are available, so one assumes the full classification of finite simple groups and considers all groups on the list. The result is included in Table PE of Section 3.

For each exceptional group, a short list of candidate finite simple groups is obtained and the focus shifts to proving existence of embeddings. For most putative embeddings in $E_{8}(\mathbb{C})$, an existence proof is easy by embedding in a suitable subgroup of the form $S L(n, \mathbb{C})(n \leq 9)$ or $S O(n, \mathbb{C})(n \leq 16)$ modulo a group of scalars. Existence of such an embedding is settled routinely with character theory and power maps as in Section 1. This method gives projective embeddings of, for example, low degree alternating groups and of $P S L(2, q)$, for certain $q \leq 29$. In a very few cases, one uses character theory to deduce existence of an anticommutative algebra structure which satisfies the Jacobi identity (easy example: the three dimensional representation of $A l t_{5}$ embeds in the adjoint group $P S L(2, \mathbb{C})$; less easy: the embedding of ${ }^{3} D_{4}(2)$ in $\left.F_{4}(\mathbb{C})[\mathrm{CW} 95]\right)$.

The hard cases are all instances of Lie primitive embeddings in an exceptional group. Most of these existence proofs involve computers, and all classifications of such embeddings done so far have required computers. Computer work here is roughly speaking more difficult as the dimension of the smallest irreducible module increases. In all these computer cases, one works over a suitable finite field, then lifts the result to characteristic 0 . For example, generators for $\operatorname{PSL}(2,27)$ are given as explicit degree 27 matrices over $\mathbb{F}_{547}$ [CW97] which give an embedding in $F_{4}(547)$, hence an embedding in $F_{4}(\mathbb{C})$. Degree 56 matrices over $\mathbb{F}_{5}$ which generate a $P S U(3,8)$ subgroup of $E_{7}(5)$ can fit on a single journal page [GRU]. The computational complexity does become significant for work in 248 dimensions. This became clear when we were able to classify embeddings of $P G L(2,31)$ but not $P S L(2,31)$ in $E_{8}(\mathbb{C})[G R 31]$. By dropping only to an index 2 subgroup, the space of parameters over $\mathbb{F}_{2791}$ that we need to search becomes too big.

A theory of Serre [S96] proves that an arbitrary simple algebraic group does contain certain $P S L(2, q)$ subgroups. The general applicability of this technique is attractive, and it gives the first existence proofs for some embeddings and alternate proofs for others that originally used a computer. The subject of [S96] is the case when $q=m h+1$ where $m \geq 1, h$ is the Coxeter number and $q$ is prime. The main cases are $m \in\{1,2\}$, for which Serre proves $P G L(2, q)$ embeds in $G$ when $m=1$ and $P S L(2, q)$ embeds in $G$ when $m=2$. He gives the first proof of one of the hard embedding cases, $P G L(2,31)$ in $E_{8}(\mathbb{C})$; later computer work [GR31] classifies equivalence classes of embeddings of $P G L(2,31)$ (there are three, two of which are given by [S96]). Serre's theory [S96] also establishes embeddings of $P S L(2,61)$ in $E_{8}(\mathbb{C}), P S L(2,19)$ and $P S L(2,37)$ in $E_{7}(\mathbb{C})$ and $P S L(2,13)$ and $P S L(2,7)$ in $G_{2}(\mathbb{C})$ (there are several old proofs of these last two embeddings). The case $m=2$ 
addresses the 1983 theory of Kostant. For an exceptional group, with Coxeter number $h$, the number $q=2 h+1$ is a prime power. Kostant noticed elements in the exceptional group which have orders $h, h+1$ and $2 h+1$ and which have traces just 0 or \pm 1 on all fundamental modules. He felt that conjugates of such elements ought to be in a finite subgroup $L \cong P S L(2, q)$ (this is for the adjoint version of the exceptional group). His conjecture was verified over the years, case by case; the constructions of [S96] are uniform and seem to give finite subgroups with the right elements, but we have not checked this. About 1994, Serre called attention to certain "Springer numbers", $n$, which come up in invariant theory [Spr]. For $E_{8}(\mathbb{C})$, besides the Coxeter number $n=30$, we have $n=20,24$. Serre felt that there might be $P S L(2,2 n+1)$ subgroups. In unpublished work, Serre has given embedding proofs for $P S L(2,41)$ in $E_{8}(\mathbb{C})$ and $P S L(2,29)$ in $E_{7}(\mathbb{C})$; see also [GR41]. Arjeh Cohen noticed this possibility for the Springer numbers but did not propose it since $[\mathrm{CG}]$ had erroneously eliminated the relevant $P S L(2,2 n+1)$ embeddings. It is interesting that " $h+1$ " for one exceptional group may be " $2 h+1$ " for another, so it may be worth studying the whole system of embeddings for a given $\operatorname{PSL}(2, q)$ in all the exceptional groups.

The theory of Sepanski [Sep] should be mentioned since it gives a uniform treatment of (previously known) embeddings of $\operatorname{PSL}(2, q)$ into the automorphism groups of the simple rank 2 Lie algebras. Let $q$ be an odd prime power. The complex irreducibles of $L:=P S L(2, q)$ have dimensions $1,(q \pm 1) / 2, q$ and $q \pm 1$. Sepanski studies certain irreducible modules $M$ of dimension $q+1$ and is able to analyze when there is an $L$-invariant Lie algebra structure on $M$. In a Lie algebra, there are integrality conditions on structure constants, and these lead to an interpretation of $(q-1) / 2$ as the Coxeter number for the Lie algebra, whence limits on $q$. The result is the set of embeddings in rank 2 groups: $P S L(2,7)$ in $A_{2}(\mathbb{C}), P S L(2,9)$ in $B_{2}(\mathbb{C})$ and $P S L(2,13)$ in $G_{2}(\mathbb{C})$. A Lie algebra structure on a module corresponds to a degree 3 invariant with the right properties; the Jacobi identity can rarely be verified with straight character theory. What is special in [Sep] is that the low degree invariants of $M$ are accessible for the relevant values of $q$, though sophisticated techniques are used.

\section{Related CLASSifications}

The Lie primitive concept (defined in Section 3) was inspired by that of a primitive linear group (Definition: a finite subgroup of $G L(n, \mathbb{C}$ ) which permutes the summands of a nontrivial direct sum decomposition is called imprimitive; otherwise primitive.) For instance, a dihedral subgroup of order at least 6 in $G L(2, \mathbb{C})$ is irreducible, but imprimitive. For a finite subgroup of $G L(n, \mathbb{C})$, Lie primitivity implies primitivity as a linear group which in turn implies irreducibility, but not conversely (e.g., take Alt $_{5}<S O(3, \mathbb{C})<S L(3, \mathbb{C})$ ).

The next result clearly shows the significance of Lie primitivity and further justifies the classification of finite quasisimple subgroups (for convenience the algebraic group below is of adjoint type, so any finite quasisimple group which arises is actually simple). Recall that the socle of a finite group is the product of its minimal normal subgroups; write $\operatorname{Soc}(F)$ for the socle of $F$.

Theorem (Borovik [Bor89][Bor90][CG93]; see also [FrG]). Let $F$ be a finite Lie primitive subgroup of the simple algebraic group $G$, adjoint type. Then one of the following holds. 
(a) There is a nontoral elementary abelian p-group $A$ so that $F \leq N_{G}(A)$, a finite Lie primitive group (all such were classified by Alekseevski [Alek]).

(b) $\operatorname{Soc}(F)$ is a finite simple group.

(c) $G=E_{8}(\mathbb{C})$, and $F \leq B$ where $\operatorname{Soc}(B) \cong A l t_{5} \times A l t_{6}$ and $B / \operatorname{Soc}(B) \cong 2 \times 2$. We call this group $B$ the Borovik group; it is unique up to conjugacy.

The Borovik group was a fascinating new discovery. A different terminology (Jordan subgroups) is used in [Alek] for the elementary abelian groups in (a). Many of them come from the following situation. Let $P$ be an extraspecial $p$-group, that is, a nonabelian $p$-group such that $P^{\prime}=Z(P)$ has order $p$; there is an integer $n>0$ so that $|P|=p^{1+2 n}$. All faithful irreducible representations are embeddings into $G L\left(p^{n}, \mathbb{C}\right)$. The image of $P$ in $P G L\left(p^{n}, \mathbb{C}\right)$ is elementary abelian and is rather obviously not toral since $P$ is nonabelian. There is a pleasant example of a nontoral elementary abelian 2-group of order 8 in $G_{2}(\mathbb{C})$ which comes as a group of sign changes with respect to a famous basis of the Cayley numbers [Cox] [GrElAb]. This $G_{2}(\mathbb{C})$-example can also be realized by a general method for constructing a nontoral elementary abelian 2-group. In a connected, simply connected quasisimple complex Lie group, we take the subgroup generated by elements of order 2 in a maximal torus and an element of order 2 in the torus normalizer that maps to -1 in the Weyl group, if such an element exists. This procedure has analogues for the primes 3 and 5 [GrElAb].

Borovik's Theorem indicates a role played by finite quasisimple subgroups of Lie groups. It has no real effect on the program to classify finite quasisimple subgroups of exceptional groups (other than providing the example (c)), but it can be useful for studying other kinds of subgroups, such as direct products of finite simple groups. For example, one could use the Borovik theorem to show that the image of any embedding of $P S L(2,11) \times P S L(2,11)$ in $E_{8}(\mathbb{C})$ must lie in an $A_{4}^{2}$ type subgroup (meaning, in a closed subgroup $N$ which is the normalizer of a cyclic group of order 5 such that the components of $N^{0}$ are each isomorphic to $S L(5, \mathbb{C})$; in fact, $\left.N^{0} \cong S L(5, \mathbb{C}) \circ S L(5, \mathbb{C})\right)$.

Embeddings of nonquasisimple finite groups in exceptional algebraic groups. There has been some systematic work on classifying finite subgroups which are abelian or whose socle is a large noncentral solvable group. Assorted results classifying smallish solvable groups may be found throughout the basic classification articles [CG] [F1][F2] [F3][K].

(1) Elementary abelian p-groups. The maximal ones have been classified in nearly simple algebraic $G$, in characteristic not $p$ (definition: nearly simple means $G^{0}$ is quasisimple and $\left.C_{G}\left(G^{0}\right) \leq G^{0}\right)$. See [GrElAb], where the summary (1.18) shows that, for each prime $p$ and nearly simple group $G$, there is up to conjugacy a finite list of nontoral maximal elementary abelian $p$-groups. In these nontoral cases, the class distributions and normalizers are studied. This category includes the groups in (a) of Borovik's Theorem.

Torality of an elementary abelian group can sometimes be detected by "local tests": for example, in $E_{8}(\mathbb{C})$, one can assign a polynomial degree to an elementary abelian 2-group; this degree is at most 3, and (with one exception) such a group is toral iff the degree is at most 2 . The issues for a definitive theory on torality are tricky. See [GrElAb], especially Section 13.

(2) Mike Kantor's thesis $[K]$ contains an analysis of subgroups $F$ of $E_{8}(\mathbb{C})$ which normalize a maximal torus $T$ and have the property that $A:=T \cap F$ is a finite 
2-group and $F / A \cong P S L(2,7)$ or $S L(2,7)$. He determined the possible structures of $A$ and the $E_{8}(\mathbb{C})$-conjugacy classes of subgroups $S$ of $F, S \cong P S L(2,7)$ or $S L(2,7)$. There were interesting homological issues involving representations of $S$ over coefficients $\mathbb{Z} / 2^{n} \mathbb{Z}$. Let $F$ be a subgroup of the torus normalizer isomorphic to $P S L(2,7)$ or $S L(2,7)$. Fix a subgroup $P$ of order 7 in $F$. Let $A$ be a finite 2-subgroup of $T$ normalized by $S$ and write $A$ as a direct product of groups $A_{3} \times$ $A_{3^{\prime}} \times A_{1}$, where the factors are the maximal $P$-submodules whose every composition factor is the respective $\mathbb{F}_{2} P$-irreducible $3,3^{\prime}, 1$ of respective dimensions $3,3,1$ (the module $3^{\prime}$ is dual to the module labelled 3 ). For $k=3,3^{\prime}, A_{k}$ is a direct product of isomorphic cyclic groups. If $2^{n(k)}$ denotes the orders of the cyclic groups, then $\left|n(3)-n\left(3^{\prime}\right)\right| \leq 2$. For each $k$, there exist subgroups in the $E_{8}(\mathbb{C})$-torus normalizer of the shape

$$
\left[\mathbb{Z}_{2^{k}}^{3} \times \mathbb{Z}_{2^{k+2}}^{3^{\prime}}\right] P S L(2,7)
$$

but none of shape

$$
\left[\mathbb{Z}_{2^{k}}^{3} \times \mathbb{Z}_{2^{k+3}}^{3^{\prime}}\right] P S L(2,7) .
$$

Connections with positive characteristic. A comparable study of projective embeddings of finite simple groups into exceptional algebraic groups of positive characteristic has been under way since the mid 80s. In positive characteristic, there are more simple groups which embed; the obvious ones are groups of Lie type over finite subfields, but also we get the first Janko group $J_{1}$ in $G_{2}(11)[\mathrm{J}]$ and the Rudvalis sporadic simple in $E_{7}(5)$ [GRU], among others. In the case where the positive characteristic divides the order of the finite group, we expect the classification of embeddings to be much more complicated than in the characteristic zero case. We do not attempt to survey the results here, but make an important point below in the coprime characteristic case. This is relevant to classifying embeddings in characteristic 0 , because we prefer computer work over a finite field.

Let $p$ be a prime number not dividing the order of the finite group $F$. The classic theory of Richard Brauer says that the reduction $\bmod p$ process gives a bijection of irreducible modules over algebraically closed fields in characteristics 0 and $p$. Here is a sketch. To reduce from characteristic 0 to characteristic $p$, one writes the representation of $F$ over a ring which is finitely generated over $\mathbb{Z}$, then reduces by a prime ideal which contains $p$. The inverse process involves creating an appropriate discrete valuation ring. One has a representation of $F$ in characteristic $p$, and it may be assumed to be written over a finite field $k$. Take a complete discrete valuation ring $R$ such that $R / \operatorname{rad}(R) \cong k$. By coprimeness, the representation may be lifted modulo increasing powers of $\operatorname{rad}(R)$. The completeness of $R$ and an inverse limit process allows us to write a representation of $F$ over $R$ which reduces modulo $R$ to the original representation over $k$. An embedding of $R$ in the complex numbers finishes the lifting.

This theory for $G L(n, \cdot)$ has an analogue for $G(\cdot)$, a Chevalley group functor. Let $F$ be a finite group. For $p$ a prime not dividing $|F|$, conjugacy classes of embeddings of $F$ in $G(\mathbb{C})$ and in $G\left(\overline{\mathbb{F}}_{p}\right)$ are in bijection by a "reduction mod $p$ " procedure. This is a very nice recent result of Michael Larsen which we call the $(0, p)$ Equivalence Theorem for coprime characteristic embeddings. An earlier version of this assuming Lie primitivity was obtained by Griess and Ryba; see [GR31] for both. Larsen's proof uses nontrivial algebraic geometry; one would hope for a more elementary proof. 


\section{REFERENCES}

[Alek] A. V. Alekseevski, Finite commutative Jordan subgroups of complex simple Lie groups, Functional Analysis Appl. 8 (1974), 277-279. MR 52:653

[Bor89] A. Borovik, On the structure of finite subgroups of the simple algebraic groups, Algebra and Logic 28 (1989), 249-279 (in Russian). MR 91h:20071

[Bor90] A. Borovik, Finite subgroups of simple algebraic groups, Soviet Math. Dokl. 40, 570573, (translation). MR 90m:20051

[BS] A. Borel and J.-P. Serre, Sur certains sous-groupes de Lie compacts, Comment. Hath. Helv., 27(1953), 128-139. MR 14:948d

[Car] R. W. Carter, Simple Groups of Lie Type, John Wiley and Sons, London, 1989. MR 90g:20001

[CG] Arjeh Cohen and Robert L. Griess, Jr, On finite simple subgroups of the complex Lie group of type $E_{8}$, Proc. Symp. Pure Math. 47 (1987), 367-405. MR 90a:20089

[CG93] Arjeh Cohen and Robert L. Griess, Jr, Non-Local Lie primitive subgroups of Lie groups, Can. J. Math. 45 (1)(1993), 88-103. MR 94a:22012

[CGL] Arjeh Cohen, Robert Griess and Bert Lisser, The group $L(2,61)$ embeds in the Lie group of type $E_{8}$, Comm. in Algebra, 21(6), 1889-1907 (1993). MR 94m:20041

[CS] Arjeh M. Cohen and Gary M. Seitz, The $r$-rank of the groups of exceptional Lie type, Nederl. Akad. Wetensch. Indag. Math. 49 (1987), no. 3, 251-259. MR 88k:20062

[CW83] Arjeh Cohen and David Wales, Finite subgroups of $G_{2}(\mathbb{C})$, Comm. Algebra 11 (1983), 441-459. MR 85b:20010

[CW93] Arjeh Cohen and David Wales, Embeddings of the group $L(2,13)$ in groups of Lie type $E_{6}$. Israel Journal of Mathematics, 82 (1993), 45-86. MR 94h:20020

[CW95] Arjeh Cohen and David Wales, Finite simple subgroups of semisimple complex Lie groups - a survey, from Groups of Lie Type and their Geometries, edited by W. M. Kantor and L. Di Martino, Cambridge University Press 1995. MR 96e:20021

[CW97] Arjeh Cohen and David Wales, On finite subgroups of $F_{4}(\mathbb{C})$ and $E_{6}(\mathbb{C})$, Proc. London Math. Soc., (3) 74 (1997), 105-150. MR 97k:20078

[Cox] Coxeter, H. S. M., Integral Cayley numbers, Duke Math. J. 13 (1946), 561-578. MR 8:370b

[Fe] Walter Feit, Characters of Finite Groups, Benjamin, New York, 1967. MR 36:2715

[FoG] P. Fong and R. Griess, An infinite family of elementwise conjugate nonconjugate homomorphisms, International Mathematics Research Notices, 1995, 5, 249-252. MR 96e:20029

[F1] Darrin Frey, Conjugacy of alternating groups of degree 5 and $S L(2,5)$ subgroups of the complex Lie group of type $E_{8}$, Thesis, University of Michigan, 1995.

[F2] Darrin Frey, Conjugacy of alternating groups of degree 5 and $S L(2,5)$ subgroups of the complex Lie group of type $E_{8}$, Memoirs of the American Mathematical Society, to appear.

[F3] Darrin Frey, Conjugacy of alternating groups of degree 5 and $S L(2,5)$ subgroups of the complex Lie group of types $F_{4}$ and $E_{6}$, to appear in Journal of Algebra.

[FrG] D. Frey and R. Griess, The conjugacy classes of elements in the Borovik group, Journal of Algebra 203 (1998), 226-243. CMP 98:12

[Frob] G. Frobenius, Über die cogredienten Transformationen der bilinearen Formen, S.-B. Preuss. Akad. Wiss. (Berlin) 7-16 (1896); Gesammelte Abhandlungen, II , 695-704.

[Gor] Daniel Gorenstein, Finite Groups, Harper and Row, New York, 1968. MR 38:229

[GrElAb] Robert L. Griess, Jr., Elementary Abelian p-Subgroups of Algebraic Groups, Geom. Dedicata 39 (1991), 253-305. MR 92i:20047

[GrG2] Robert L. Griess, Jr., Basic conjugacy theorem for $G_{2}$, Invent. Math., 121 (1995) 257-277. MR 96f:20065

[GrTwelve] Robert L. Griess, Jr., Twelve Sporadic Groups, Springer Mathematical Monograph, Springer Verlag, 1998.

[GRU] Robert L. Griess, Jr. and A. J. E. Ryba, Embeddings of $U(3,8), S z(8)$ and the Rudvalis group in algebraic groups of type $E_{7}$, Inventiones Math. 116, 215-241 (1994). MR 94k:20024

[GR31] Robert L. Griess, Jr. and A. J. E. Ryba, Embeddings of $P G L(2,31)$ and $S L(2,32)$ in $E_{8}(\mathbb{C})$, Duke Math. Journal 94 (1998), 181-211. CMP 98:16 
[GR41] Robert L. Griess, Jr. and A. J. E. Ryba, Embeddings of $P S L(2,41)$ and $P S L(2,49)$ in $E_{8}(\mathbb{C})$, to appear in Journal of Symbolic Computation.

[GRQ] Robert L. Griess, Jr. and A. J. E. Ryba, The finite quasisimple groups which embed in exceptional Lie groups. Preprint.

[GR8] Robert L. Griess, Jr. and A. J. E. Ryba, Embeddings of $S z(8)$ into exceptional Lie groups. Preprint.

[Hup] B. Huppert, Endliche Gruppen I, Springer Verlag, Berlin, 1968. MR 37:302

[I] I. Martin Isaacs, Character Theory of Finite Groups, Academic Press, 1976. MR $\mathbf{5 7 : 4 1 7}$

[J] Zvonimir Janko, A new finite simple group with abelian Sylow 2-subgroups and its characterization, J. Algebra 3 147-186 (1966). MR 33:1359

$[\mathrm{K}] \quad$ Michael J. Kantor, $S L(2,7)$ and $P S L(2,7)$ Subgroups of $E_{8}(\mathbb{C})$ and their Actions on a Maximal Torus, Thesis, University of Michigan, 1996.

[KR] P. Kleidman and A. J. E. Ryba, Kostant's Conjecture Holds for $E_{7}: L_{2}(37)<E_{7}(\mathbb{C})$, J. Alg. 161(2), 535-540 (1993). MR 94k:20025

[Kul] B. Kulshammer, Algebraic representations of finite groups, Universität Augsburg, 1992.

[Lar1] M. Larsen, On the conjugacy of element-conjugate homomorphisms, Israel Journal, 88 (1994), 253-277. MR 95k:20073

[Lar2] M. Larsen, On the conjugacy of element-conjugate homomorphisms, II, Quart. J. Math. Oxford Ser. (2) 47 (1996), 73-85. MR 97b:20070

[Mal] A. I. Mal'cev, Semisimple subgroups of Lie groups, Amer. Math. Soc. Translations 1, 172-273 (1962).

[McK] John McKay, Finite Groups - Coming of Age, Proc. Can. Math. Soc. Conf., held June 15-28, 1982, Amer. Math. Soc., Providence, RI, 1985, 271-285. MR 86j:20002

[Sep] Mark R. Sepanski, Kostant's conjecture and $L_{2}(q)$ invariant theory in the rank two Lie groups, Comm. Alg., 24 (1996), no. 6, 1915-1938. MR 97g:20020

[S96] J-P. Serre, Exemples de plongements des groupes $P S L\left(2, \mathbb{F}_{p}\right)$ dans des groupes de Lie simples, Inventiones Math. 124, 525-562 (1996). MR 97d:20056

[SP] J-P. Serre, Personal Communication, 1998.

[Slo] Peter Slodowy, Two notes on a finiteness problem in the representation theory of finite groups, Hamburger Beiträge zur Mathematik, aus dem Mathematischen Seminar, Heft 21; 1993. Published in "Algebraic Groups and Lie Groups" (A volume of papers in honour of the late R.W. Richardson), Ed. G.I. Lehrer, Australian Math. Soc. Lecture Series No. 9, Cambridge University Press, Cambridge, 1997; pages 331-346. CMP 98:16

[Spr] T. A. Springer, Regular elements of finite reflection groups, Inventiones Math. 25 (1974), 159-198. MR 50:7371

[Tits55] Jacques Tits, Sous-algèbres des algèbres de Lie semi-simples (d'apres V. Morosov, A. Malcev, E.Dynkin et F. Karpelevitch), Séminaire Bourbaki, No. 119, May 1955. CMP 98:09

[W] David Wales, Finite linear groups of degree 7, Canadian J. Math. 21, 1025-1041 (1969) [see also Bull. Amer. Math. Soc. 74, 197-198 (1968). MR 40:1489

[Weil] A. Weil, Remarks on the cohomology of groups, Ann. Math. 80 (1964), 149-157; also [1964a], vol.3 in Oeuvres scientifiques ; Collected papers / Andre Weil. New York : Springer-Verlag (1979), 3 volumes. MR 30:199

Department of Mathematics, Univerity of Michigan, Ann Arbor, Mi 48109-1003

E-mail address: rlg@math.lsa.umich.edu

Department of Mathematics, Marquette University, Milwaukee, WI 53201-1881

Current address: Department of Mathematics, Queens College, CUNY, Flushing, NY 113671597

E-mail address: alexr@sylow.mscs.mu.edu 